Original Article

\title{
Greater social jetlag associates with higher HbA1c in adults with type 2 diabetes: a cross sectional study
}

\author{
Rachael M. Kelly a, Jacinta Finn a, Ultan Healy ${ }^{\mathrm{b}}$, Dervla Gallen ${ }^{\mathrm{a}}$, Séamus Sreenan b, c, \\ John H. McDermott ${ }^{\mathrm{b}, \mathrm{c}}$, Andrew N. Coogan ${ }^{\mathrm{a}, *}$ \\ a Department of Psychology, Maynooth University, National University of Ireland, Maynooth, Ireland \\ ${ }^{\mathrm{b}}$ Academic Department of Endocrinology, Royal College of Surgeons in Ireland, Connolly Hospital Blanchardstown, Dublin, Ireland \\ c $3 U$ Diabetes Consortium, Dublin, Ireland
}

\section{A R T I C L E I N F O}

\section{Article history:}

Received 30 April 2019

Received in revised form

2 July 2019

Accepted 12 July 2019

Available online 5 August 2019

\section{Keywords:}

Chronotype

Social jetlag

Personality

Glycemic control

HbA1c

Type 2 diabetes

\begin{abstract}
A B S T R A C T
Background/Objectives: Later chronotype has been associated with poorer glycemic control in type 2 diabetes. It is unclear whether this is a direct relationship, or if personality factors or social jetlag ([SJL], ie, chronic circadian misalignment reflecting the discrepancy between the entrained phase of the circadian clock and socially-determined behavioural cycles) play a role. This study aimed to determine the relationships among chronotype, SJL, personality factors and glycemic control in type 2 diabetes, independently of sleep disturbances and daily caloric distribution.

Methods: In sum, 252 type 2 diabetes patients attending an annual review outpatients' clinic completed questionnaires, including the Munich Chronotype Questionnaire to assess chronotype and SJL, the Pittsburgh Sleep Quality index (PSQI), the Big Five Personality Inventory and the Center for Epidemiologic Studies Depression Scale. Chart review provided information on diabetes duration, Hemoglobin A1C (HbA1c), body mass index (BMI) and other clinical variables. Caloric intake was assessed via 24-h dietary recall.

Results: Hierarchical linear regression revealed that SJL, but not chronotype or personality factors, was a significant predictor of HbA1c levels $(\beta=0.16, p<0.05)$. There was a significant relationship between later chronotype and HbA1c levels, but only in patients who had more than 90 min SJL $(r=0.51$, $\mathrm{p}=0.002)$. Younger age was associated with a higher $\operatorname{HbA1c}(\mathrm{r}=-0.23, \mathrm{p}<0.001)$, and this effect was partially mediated through SJL $(\mathrm{Pm}=0.22)$.

Conclusions: We identify SJL as a novel factor that may impact on glycemic control in type 2 diabetes. Further study is needed to determine whether interventions aimed at reducing SJL may lead to improvements in glycemic control.
\end{abstract}

(ㄷ) 2019 Elsevier B.V. All rights reserved.

\section{Introduction}

Circadian clocks are fundamental to the daily temporal architecture of physiology and metabolism, with circadian rhythms (ie. those with a period of near $24 \mathrm{~h}$ ) emerging as products of an endogenous biological timekeeping system [1]. Inter-individual differences in circadian processes and entrainment phase manifests behaviourally as differences in actual and/or preferred sleep and wake time, referred to as chronotype [2,3]. Modern 24-h

\footnotetext{
* Corresponding author. Department of Psychology, Maynooth University, County Kildare, Ireland.

E-mail address: andrew.coogan@mu.ie (A.N. Coogan).
}

society promotes activity and behaviours which may be mistimed relative to the circadian cycle, resulting in misalignment between 'internal' biological time and 'external' social time. The potential negative consequences of circadian misalignment on cardiometabolic functioning are evident from studies of the associations of shift work (which results in significant circadian misalignment) with increased risk and prevalence of type 2 diabetes $[4,5]$ and obesity [6].

A prevalent form of circadian misalignment that arises as a function of the conflicting interaction between intrinsic circadian biology and social/work obligations is social jetlag (SJL). SJL has a specific quantitative definition as the difference between the midpoint of sleep time on "free" days (those days free from sociallyimposed influences on timing of sleep/wake, and therefore on 
which sleep timing is predominantly shaped by the circadian clock) and work days (on which sleep timing is more strongly shaped by social imperatives) [7]. As such, SJL is partly a function of an individuals' chronotype which predicts approximately 50\% of the variation of SJL in young adults [8]. As modern society broadly favours morning orientation, later chronotype is associated with greater degrees of SJL [8]. Furthermore, as chronotype is strongly influenced by age (with late chronotype peaking in the early twenties), SJL is most marked in late adolescence and early adulthood $[2,8]$. SJL has been proposed as a risk factor for a number of pathologies and pathophysiological conditions, including type 2 diabetes $[9,10]$ and elevated body mass index (BMI) $[8,11]$.

A number of previous studies have examined chronotype and disease outcomes in type 2 diabetes. Reutrakul et al. [12], reported an association between later chronotype and Hemoglobin A1c (HbA1c) levels in individuals with type 2 diabetes, independent of other sleep and clinical variables. This association was partially mediated by breakfast skipping [13]. Later chronotype and higher HbA1c levels have also been associated in a prediabetes cohort [14], indicating that metabolic dysregulation in these conditions may be influenced by the phase of circadian entrainment in every-day life. There is also a relationship between diurnal preference and BMI in type 2 diabetes, which is partially mediated by timing of breakfast [15]. Research on breakfast skipping and long-term metabolic health continues to produce mixed results [16], with some reports of strong associations between breakfast skipping and higher postprandial hyperglycemia, resulting in higher HbA1c [17]. With regards to SJL, an association between SJL, higher fasting glucose and increased risk of obesity and resulting metabolic complications has been identified among individuals with non-communicable chronic disorders [18], although in type 2 diabetes one study showed no relationship between SJL and HbA1c [12]. Parsons et al., reported that individuals belonging to the metabolically unhealthy obese sub-group with higher SJL were more likely to have higher HbA1c levels; however this association did not persist after controlling for smoking and socioeconomic status [11]. SJL was reported to be associated with higher HbA1c levels in a type 1 diabetes population [19], although another recent study found no relationship between HbA1c and either SJL or chronotype in an adolescent population with type 1 diabetes (SJL was however associated with a requirement for insulin in this cohort [20]). However, recent research failed to identify any association between SJL or chronotype and measures of glycemia in adults with prediabetes and recently diagnosed untreated type 2 diabetes [21].

From a behavioural/psychological perspective, the impact of chronotype on (patho-) physiology may be mediated through its relationship with various psychological domains [22]. Chronotype is associated with personality factors, with evening orientation associated with higher extraversion and openness, and morning orientation associated with higher conscientiousness and agreeableness [22,23]. Conscientiousness may also be associated with lower obesity and better self-care behaviours in patients with type 2 diabetes [24]; thus it is possible that the association of earlier chronotype with better glycemic control could be mediated through conscientiousness. While SJL is not wholly collinear with chronotype, individuals with a later chronotypes are more likely to have more SJL [8]; it is therefore possible that SJL is also associated with these psychological domains. However, no clear understanding of the possible direct relationship between SJL and personality traits exists, although any association between SJL and glycemic control may be understood from a behavioural perspective as the result of numerous social and psychological factors influence diabetes management [25]. SJL and chronotype have been demonstrated to have an impact on the amount of some food groups consumed at different times of the day [26]. Furthermore, SJL may influence food behaviour in a negative manner. Recent research demonstrated an association between later meal times, poorer diet and SJL [27]. Therefore, behaviours that SJL elicits could be crucial in understanding its role glycemic control.

Given the preceding context indicating potential clinical significance of the timing of sleep/wake behaviour in type 2 diabetes, we set out to determine if chronotype and/or SJL were associated with glycemic control independently of other sleep disturbances and psychological domains in a cohort of adults with type 2 diabetes. We hypothesised that chronotype would be associated with glycemic control, and that SJL and personality may partially mediate such an association. The ultimate goal is to identify potential chronobiological substrates that may be tractable for behavioural interventions in the clinical management of type 2 diabetes.

\section{Materials and methods}

\subsection{Participants}

In total, 252 adult patients with type 2 diabetes attending the outpatients annual review clinic at the Diabetes Centre, Connolly Hospital Blanchardstown, Dublin (169 males and 83 females) were recruited for this study. Sample size was estimated based on a previous study in the area [12] and the sample size required for the regression analysis anticipated prior to data collection. Guidelines were followed and a recommended formula to calculate the minimum sample required $(\mathrm{N}>50+8 \mathrm{~m}$; where $\mathrm{m}$ is the number of predictor variables) [28]. In our case 252 was greater than 138 $[50+8(11)]$ and also accounted for skew in HbA1c as the dependent variable, even after log-transformation. Individuals attending their scheduled appointment at the clinic were approached in the waiting room. This clinic ran for 4-5 h every week and patients were asked if they would like to participate upon arrival. If individuals were interested, they were screened to see if they met the inclusion and exclusion criteria and given an information leaflet. The inclusion criteria were age of greater than 18 , a diagnosis of type 2 diabetes in the absence of serious diabetes complications and medical co-morbidities, and an ability to provide informed consent. Shift-workers were excluded. Mean patient age was 61.9 years (SD $=10.5$, range $31-87$ ). Informed consent was obtained from all participants and they were then provided with a questionnaire booklet and the dietary recall. Participants either filled this out by themselves under the supervision of the researcher or the researcher assisted them with the questionnaires. The study was approved by the Research Ethics Committee of Connolly Hospital and conformed to the Declaration of Helsinki.

\subsection{Assessments}

Participants' age, weight, BMI, duration of diabetes, current medications, use of insulin and most recent HbA1c levels were obtained from chart review. The participants attended a preassessment clinic two weeks prior to their appointment which is when the HbA1c is assessed. There was therefore a two-week lapse between HbA1c determination and the date that questionnaires were completed, an appropriate timeframe given that HbA1c is a reliable indicator for blood glucose control over a three month period. Participants completed one 24 -h dietary recall by providing a description of the content, and quantity of meals and snacks consumed in the previous $24 \mathrm{~h}$. They were asked to provide details regarding portion sizes and ingredients and were encouraged to provide brand names where possible. Caloric content was then calculated for overall daily consumption and for meals which were classed as breakfast, lunch, dinner or snacks. Online databases (www.myfitnesspal.ie; www.nutracheck.co.uk) and restaurant and 
manufacturer websites were used to provide information on nutritional content. These databases have accurate caloric information for standard measures of all generic foods and where specific examples and brands were not available the restaurant and manufacturer websites provide accurate information.

The Munich Chronotype Questionnaire (MCTQ) [29] was used to provide an estimate of participants' chronotype and SJL. This instrument asks participants to indicate their sleep and wake times on both "work" and "free" days, allowing for the calculation of midpoint of sleep (mid-sleep) on both types of day. Average sleep duration over the course of a week was calculated using a formula that weighted the amount of self-reported sleep on "work" and "free" days [8]. Sleep duration on workdays was multiplied by the number of workdays and added to sleep duration on free days multiplied by the number of free days; this value was then divided by 7 to get an average daily sleep duration [(SDw x WD + SDf x FD)/ 7] [8]. Mid-sleep on free days (the midpoint between sleep onset and wake time), corrected for sleep debt accumulated during the week, provided a measure of chronotype; sleep timing without social constraint provides an indication of the underlying phase of circadian entrainment (MSFsc) [29]. If sleep debt was present (ie, if sleep duration is longer on a free day than a work day) the difference between sleep duration on a free day and the average weekly sleep duration is calculated and divided by two; this was then subtracted from the original mid-sleep free to account for sleep debt [MSF - (SDf- SDweek)/2]. SJL was measured by subtracting mid-sleep on workdays from mid-sleep on free days and getting the absolute difference [|MSF - MSW|] [7]. The number of work and free days were also assessed through this instrument, as was whether there was any meaningful distinction between "work" and "free" days for the participant.

The Centre for Epidemiologic Studies Depression (CES-D) scale was used to assess depressive symptomatology and mood [30]. Participants were required to rate several statements regarding their mood over the previous week from rarely/none of the time (less than one day) to most/all of the time (5-7 days). Higher scores indicate more depressive symptoms with 16 and above indicating risk of clinical depression. The 44 item Big Five Personality Index was used to assess the five major personality domains; extraversion, agreeableness, conscientiousness, neuroticism and openness [31]. This multidimensional personality inventory begins with the statement "I see myself as someone who", followed by 44 short phrases that an individual rates on a five point Likert scale, where 1 corresponds to "disagree strongly" to 5 corresponding to "agree strongly". Each personality domain has a specified number of questions which dictates its range in scores, extraversion and neuroticism have eight questions meaning scores can range from 8 to 40 , agreeableness and conscientiousness have nine questions meaning scores can range from 9 to 45 and openness has ten questions meaning scores can range from 10 to 50 .

The Pittsburgh Sleep Quality Index (PSQI) [32] was used to evaluate subjective sleep quality and disturbances over the previous month, with poor sleep quality indicated by a global score greater than 5. The PSQI also gives seven component scores around subjective sleep quality, sleep latency, sleep duration, habitual sleep efficiency, sleep disturbances, use of sleeping medication and daytime dysfunction. The Berlin Questionnaire was used to assess the risk of obstructive sleep apnea (OSA) in participants without a previous diagnosis, with participants categorised as low risk, high risk, or having an existing OSA diagnosis [33].

\subsection{Statistical analyses}

For analysis of circadian variables MSFsc, average sleep duration and SJL were decimalised (ie 7:30 became 7.5, $45 \mathrm{~min}$ became 0.75 ). Data was assessed for potential violations of the assumptions of the general linear model. When assessing normality, skewness and kurtosis values with a cut off of \pm 2 were decided on, with values greater than \pm 2 indicating a substantial deviation from normality. Variables violating the assumption of normality were transformed using the common $\log$ transformation to reduce skewness and kurtosis and so improve overall distribution. Log $10 \mathrm{HbA1c}$ was used throughout. Following transformation, if a variable was still displaying a large deviation from the normal distribution, the correct nonparametric analysis was conducted and results were reported as median and interquartile range (IQR). To assess the relationship between glycemic control and demographic, personality, and dietary variables, Pearson product moment correlation was carried out with common Log HbA1c. This was also carried out with sleep and circadian variables except in the case of SJL, average sleep duration and diabetes duration where Spearman's rank order correlation was utilised. To assess any independent association between chronotype and HbA1c beyond demographic, personality and sleep variables a hierarchical multiple regression was conducted using predictor variables identified through previous univariate analysis (those with $\mathrm{P}<0.1$ for their association with $\mathrm{HbA} 1 \mathrm{c}$ ), whilst ensuring that the assumptions of multicollinearity, normality of distribution of residuals and homoscedasticity were not violated. This analysis adjusted for the demographic and mood variables already known to have an influence including age, sex, BMI, sleep apnea risk, insulin use, and diabetes duration. Mediation and moderation analysis were conducted using the PROCESS macro for SPSS [34]. For both moderation and mediation bootstrapping was used with 1000 samples. With the PROCESS tool model 1 was used to conduct simple moderation and model 4 was used to conduct simple mediation. All statistical analysis was conducted using IBM SPSS (V25, IBM Corporation) or JASP (V 0.9.1.0, https://jasp-stats.org/).

\section{Results}

Clinical and demographic characteristics of the 252 participants are detailed in Table 1 . A total of $62.2 \%$ of patients were obese, of whom $25.5 \%$ had severe obesity (BMI $\geq 35 \mathrm{~kg} / \mathrm{m}^{2}$ ), $32.7 \%$ were overweight and $5.2 \%$ were normal weight. $16.3 \%$ of patients were currently using insulin. Median diabetes duration was seven years and median HbA1c level was $6.9 \%$ (52 mmol/mol). Analysis of circadian variables showed that the average midsleep on free days was 3:53 am while SJL displayed a large range (0-304 min; median $=15 \mathrm{~min}$ ). Good subjective sleep quality was evident in $51 \%$ (reflected by a global PSQI score of 5 or less). OSA risk was evident in $50.4 \%$ of the population, while $2.4 \%$ reported a diagnosis; due to the small number of people with an OSA diagnosis they were grouped in with the individuals at high risk and OSA was analysed as a dichotomous variable.

Correlation analysis of log transformed HbA1c and demographic, dietary, personality and circadian and sleep variables are displayed in Table 2. Higher HbA1c levels were associated with more depressive symptoms, longer disease duration, higher BMI and younger age. Total daily calories, or calories consumed at breakfast, lunch and dinner, were not associated with HbA1c levels (see Table 2). Higher HbA1c was associated with lower conscientiousness $(\mathrm{r}=-0.13, \mathrm{p}=0.041)$ and higher neuroticism $(\mathrm{r}=0.19, \mathrm{p}=0.003)$ but did not show a significant relationship with other personality variables. There were no statistically significant associations between sleep quality or average sleep duration and HbA1c levels. HbA1c and MSFsc were not significantly correlated, although HbA1c was significantly positively correlated with SJL (rho $=0.23, \mathrm{p}<0.001$ ). 
Table 1

Demographic, clinical, sleep and personality descriptives for the study sample.

\begin{tabular}{|c|c|}
\hline & $\mathrm{n}=252$ \\
\hline \multicolumn{2}{|l|}{ Clinical Parameters } \\
\hline Age (years) & $61.85 \pm 10.54$ \\
\hline Male ( $\mathrm{n} \%)$ & $169(67.1)$ \\
\hline BMI $\left(\mathrm{kg} / \mathrm{m}^{2}\right)$ & $32.12 \pm 5.37 n=251$ \\
\hline Normal weight & $13(5.2 \%)$ \\
\hline Overweight & $82(32.7 \%)$ \\
\hline Obesity class I & $92(36.7 \%)$ \\
\hline Obesity class II & $64(25.5 \%)$ \\
\hline Duration of diabetes (years) & $7(4-11.5) \mathrm{n}=232$ \\
\hline$<\mid=5$ & $79(34.1)$ \\
\hline $6-10$ & $90(38.8)$ \\
\hline $11-20$ & $56(24.1)$ \\
\hline$>20$ & $7(3.0)$ \\
\hline Insulin use (n, \%) & $41(16.3 \%) \mathrm{n}=251$ \\
\hline $\mathrm{HbA} 1 \mathrm{c}(\mathrm{mmol} / \mathrm{mol})$ & $52(46-62)$ \\
\hline $\operatorname{HbA1c}(\%)$ & $6.9(6.4-7.8)$ \\
\hline CES-D Score & $10.01(9.31) \mathrm{n}=240$ \\
\hline \multicolumn{2}{|l|}{ Circadian Parameters } \\
\hline Corrected MidSleep (MSFsc) (h) & $3.88(1.26)$ \\
\hline Absolute Social Jetlag (decimialised mins) & $0.25(0.00-0.95)$ \\
\hline \multicolumn{2}{|l|}{ Sleep Parameters } \\
\hline Average sleep duration & $7.62(6.95-8.5)$ \\
\hline Global sleep quality & $5.99(3.66) \mathrm{n}=249$ \\
\hline PSQI score $>5$ & $122(49 \%)$ \\
\hline Sleep onset workdays & $23.70(1.50)$ \\
\hline Sleep end workdays & $7.33(1.46)$ \\
\hline Sleep onset freedays & $00.09(1.48)$ \\
\hline Sleep end freedays & $8.02(1.66)$ \\
\hline Sleep apnea risk (n \%) & $132(52.8) \mathrm{n}=250$ \\
\hline Personality data & $\mathrm{n}=244$ \\
\hline Extraversion & $25.33(6.52)$ \\
\hline Agreeableness & $36.33(5.74)$ \\
\hline Conscientiousness & $35.45(6.80)$ \\
\hline Neuroticism & $21.63(6.98)$ \\
\hline Openness & $32.28(7.98)$ \\
\hline Dietary Parameters & $\mathrm{n}=157$ \\
\hline Total daily calories & $1321.89(370.73)$ \\
\hline Breakfast calories & $332.76(147.93)$ \\
\hline Lunch Calories & 319.57 (187.86) \\
\hline Dinner Calories & $472.43(188.21)$ \\
\hline
\end{tabular}

Data are means \pm SD (for normally distributed variables), median (25th percentile 75th percentile; for variables violating the assumption of normality) or $n$ (\%) (number of cases and the percentage that this is for categorical variables). BMI; Body Mass Index, CES-D; Centre for Epidemiologic Studies Depression scale, PSQI; Pittsburgh sleep quality index, OSA; Obstructive sleep apnea.

A hierarchical multiple regression analysis was undertaken to further assess the association between demographic and clinical variables (sex, age, BMI, Berlin score, duration of diabetes, insulin use and CES-D scores; model 1), personality variables (neuroticism and conscientiousness; model 2), sleep and circadian variables (SJL, MSFsc; model 3) and HbA1c levels (Table 3). Predictor variables entered were those that showed a $\mathrm{p}<0.10$ in the preceding simple regression analyses. Prior to conducting the multiple regression analysis the association between SJL and MSFsc was assessed in order to ensure that strong collinearity was not present, and a weak correlation was observed ( $\mathrm{rho}=0.267, \mathrm{p}<0.0005$; Supplementary Figure 1). Demographic variables explained $23.2 \%$ of the variance in HbA1c, with age, duration of diabetes and insulin use being significant predictors in model 1 . Addition of neuroticism and conscientiousness as predictors in block 2 did not significantly change $\mathrm{R}^{2}$. Addition of SJL and MSFsc as a predictor in model 3 increased $\mathrm{R}^{2}$ to 0.257 , with SJL (but not MSFsc) being a significant predictor. Further, running separate regression models with either SJL or MSFsc as the only predictor added in block 3 revealed the SJL, but not MSFsc, was a significant predictor of HbA1c (data not shown).

\section{Table 2}

Correlation analysis of $\log \mathrm{HbA} 1 \mathrm{c}$ with clinical factors, personality factors and sleep variables. All analyses are Pearson product moment correlations except for the relationship between log $10 \mathrm{HbA} 1 \mathrm{c}$ and average sleep duration, social jetlag and diabetes duration where Spearman's rho was utilised due to violations in the assumption of normality.

\begin{tabular}{|c|c|c|}
\hline & $\mathrm{r}$ & p-value \\
\hline \multicolumn{3}{|l|}{ Clinical Parameters } \\
\hline Age & $-0.23^{* * *}$ & 0.0003 \\
\hline$B M I$ & $0.18^{* *}$ & 0.005 \\
\hline$C E S-D$ & $0.22^{* *}$ & 0.001 \\
\hline Diabetes Duration $^{\text {a }}$ & $0.17^{*}$ & 0.01 \\
\hline \multicolumn{3}{|l|}{ Dietary Parameters } \\
\hline Total Daily Calories & 0.02 & 0.86 \\
\hline Breakfast Calories & -0.06 & 0.46 \\
\hline Lunch Calories & -0.02 & 0.78 \\
\hline Dinner Calories & -0.07 & 0.42 \\
\hline \multicolumn{3}{|l|}{ Personality Data } \\
\hline Neuroticism & $0.19^{* *}$ & 0.003 \\
\hline Conscientiousness & $-0.13^{*}$ & 0.04 \\
\hline Openness & 0.03 & 0.67 \\
\hline Agreeableness & -0.03 & 0.62 \\
\hline Extraversion & -0.03 & 0.67 \\
\hline \multicolumn{3}{|l|}{ Sleep Parameters } \\
\hline MSFsc & 0.11 & 0.09 \\
\hline$S J L^{\mathrm{a}}$ & $0.23^{* * *}$ & 0.0003 \\
\hline Sleep Quality (PSQI) & 0.03 & 0.61 \\
\hline Sleep Onset WD & 0.05 & 0.39 \\
\hline Sleep End WD & -0.06 & 0.33 \\
\hline Sleep Onset FD & 0.12 & 0.06 \\
\hline Sleep End FD & $0.14^{*}$ & 0.03 \\
\hline Average sleep duration ${ }^{a}$ & -0.08 & 0.23 \\
\hline
\end{tabular}

Pearson $r$ used correlation analysis.

BMI; Body Mass Index, CES-D; Centre for Epidemiologic Studies Depression scale, PSQI; Pittsburgh sleep quality index, OSA; Obstructive sleep apnea. SJL = Social jetlag, WD $=$ Work Day, FD = Free Day.

a Spearman rho correlation analysis.

To further probe the impact of SJL on HbA1c levels, four SJL groups [those with zero SJL $(\mathrm{N}=114)$, those with low SJL (less than 30 min; $N=57$ ), those with moderate SJL (between 30 and 90 min; $\mathrm{N}=46$ ) and those with high SJL (more than 90 min; $\mathrm{N}=35$ )] were constructed to test dose-response relationships between SJL and HbA1c. There was a significant difference in HbA1c levels between these SJL groups $\left(\mathrm{F}_{3,248}=5.7, \mathrm{p}<0.001\right)$, with Tukey post-hoc analysis demonstrating that the group with SJL of 90 min or more had higher HbA1c levels than those with no SJL $(\mathrm{p}<0.001$, Cohen's $\mathrm{d}=-0.815$; Fig. 1 ). This effect persisted when MSFsc was included as a covariate in an $\operatorname{ANCOVA}\left(\mathrm{F}_{3,247}=4.83, \mathrm{p}=0.003\right)$. There was no difference between SJL groups in BMI $(\mathrm{p}=0.056)$, average sleep duration ( $\mathrm{p}=0.383)$, CES-D score $(\mathrm{p}=0.973)$, breakfast calories $(\mathrm{p}=0.848)$ or total PSQI score $(\mathrm{p}=0.394$; Fig. 1$)$. Personality variables did not vary significantly across SJL groups (supplementary table 1). The high SJL group was significantly younger than the no and low SJL groups ( $\mathrm{P}<0.001$; supplementary table 1$)$. The duration of diabetes differed between groups $(p=0.044)$; however after Bonferroni adjusted pairwise comparisons no significant differences between groups remained. Since BMI displayed a $p$ value below 0.1 the relationship between SJL and BMI was analysed. There was no correlation between BMI and SJL (rho $=0.074$, $\mathrm{p}=0.245$; Supplementary Figure 2). Further, SJL and BMI were not correlated in any of the SJL groups.

To control for the potential confounding effect of age and diabetes duration, an ANCOVA was run on HbA1c levels and SJL group, with these two variables as covariates. The effect of SJL on HbA1c persisted when controlling for age and diabetes duration $(\mathrm{p}=0.022)$ with the high SJL group having a significantly higher HbA1c than the no SJL group ( $p=0.012$, Cohen's $d=-0.653$ ). Somewhat surprisingly, we observed an association between 
Table 3

Relative contribution of each variable toward the variance in log of HbA1c.

\begin{tabular}{|c|c|c|c|c|c|c|}
\hline \multirow[t]{3}{*}{ Variable } & \multicolumn{2}{|l|}{ Model 1} & \multicolumn{2}{|l|}{ Model 2} & \multicolumn{2}{|l|}{ Model 3} \\
\hline & \multicolumn{2}{|c|}{$\mathrm{R}^{2}=0.23^{* * *}$} & \multicolumn{2}{|l|}{$\mathrm{R}^{2}=0.23$} & \multicolumn{2}{|c|}{$\mathrm{R}^{2}=0.26^{*}$} \\
\hline & $\beta$ & $95 \% \mathrm{CI}$ & $\beta$ & $95 \% \mathrm{CI}$ & $\beta$ & $95 \% \mathrm{CI}$ \\
\hline (1) Sex & -0.02 & $-0.14 ; 0.10$ & -0.02 & $-0.15 ; 0.10$ & -0.02 & $-0.15 ; 0.10$ \\
\hline (1) Age & $-0.23^{* *}$ & $-0.36 ;-0.10$ & $-0.23^{* *}$ & $-0.36 ;-0.10$ & $-0.17^{*}$ & $\begin{array}{l}-0.31 \\
-0.03\end{array}$ \\
\hline (1) BMI & 0.05 & $-0.08 ; 0.18$ & 0.04 & $-0.09 ; 0.17$ & 0.04 & $-0.09 ; 0.17$ \\
\hline (1) Berlin & 0.04 & $-0.09 ; 0.17$ & 0.04 & $-0.09 ; 0.17$ & 0.01 & $-0.12 ; 0.15$ \\
\hline (1) Diabetes duration & $0.14^{*}$ & $0.00 ; 0.27$ & $0.14^{*}$ & $0.00 ; 0.28$ & 0.13 & $-0.00 ; 0.27$ \\
\hline (1) Insulin use & $-0.31^{* * *}$ & $-0.44 ;-0.18$ & $-0.31^{* * *}$ & $-0.44 ;-0.18$ & $-0.32^{* * * *}$ & $-0.45 ;-0.20$ \\
\hline (1) CES-D & 0.12 & $-0.00 ; 0.25$ & 0.10 & $-0.06 ; 0.25$ & 0.12 & $-0.03 ; 0.28$ \\
\hline (2) Conscientiousness & & & -0.03 & $-0.17 ; 0.10$ & 0.00 & $-0.14 ; 0.14$ \\
\hline (2) Neuroticism & & & 0.03 & $-0.13 ; 0.18$ & 0.02 & $-0.13 ; 0.18$ \\
\hline (3) SJL & & & & & $0.16^{*}$ & $0.02 ; 0.29$ \\
\hline (3) MSFsc & & & & & 0.04 & $-0.10 ; 0.17$ \\
\hline
\end{tabular}

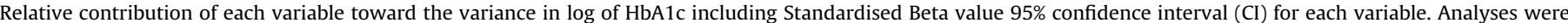

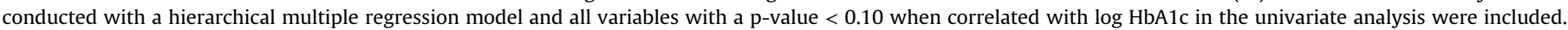
Model number at which entered is indicated for each predictor in parentheses.

${ }^{* * *} \mathrm{P}<0.001,{ }^{* *} \mathrm{P}<0.01,{ }^{*} \mathrm{P}<0.05$
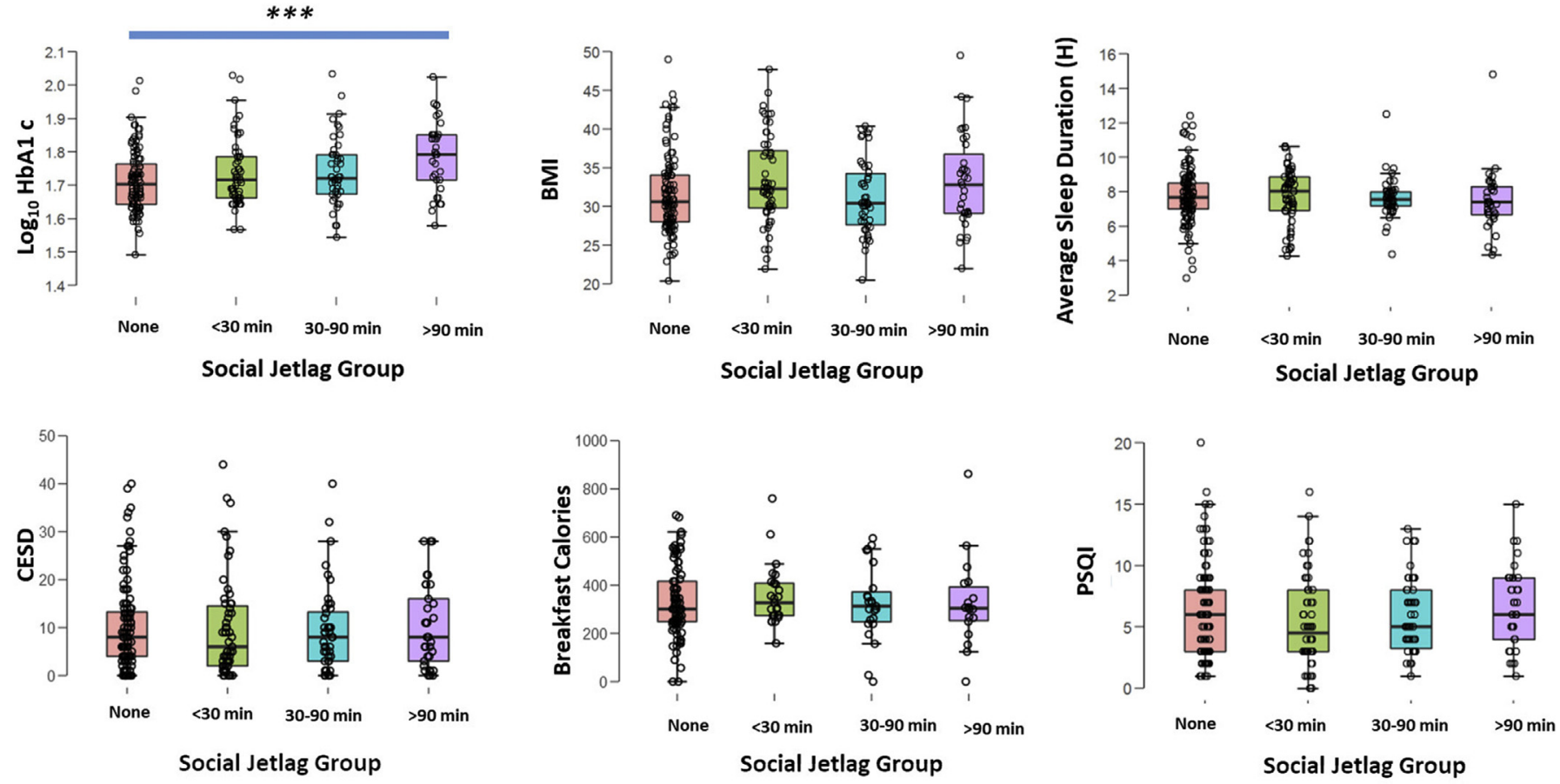

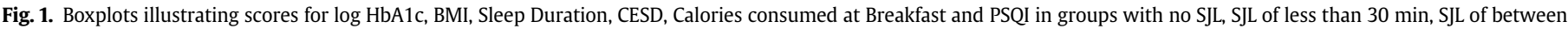

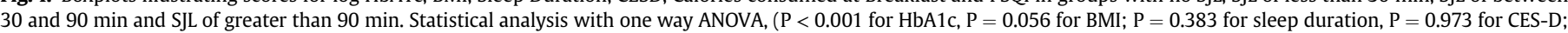

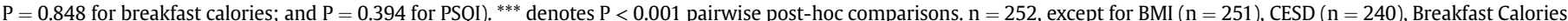
$(\mathrm{n}=157)$, PSQI $(\mathrm{n}=249)$.

younger age with higher HbA1c in the study sample $(r=-0.225$, $\mathrm{p}<0.001$ for age vs. HbA1C). As younger age is also associated with greater SJL, in our sample, we conducted a mediation analysis of the association of younger age with higher HbA1c levels, showing that this relationship is partially mediated through SJL ( $22 \%$ of the association between younger age and HbA1c levels was mediated through SJL; Fig. 2).

Given previous descriptions of associations between HbA1c and MSFsc values we performed further analysis to explore the lack of such an association in our data, and examined the association between HbA1c and MSFsc in the four SJL groups. We conducted a moderation analysis of the relationship between MSFsc and HbA1c, with SJL group as the moderator. This analysis showed that including the MSFsc $\mathrm{x}$ SJL group term increased $R^{2}$ by 0.043 , and that this interaction was significant $(p=0.001)$. These results indicate that MSFsc is associated with HbA1c levels, but only in the presence of high levels of SJL $(\mathrm{r}=0.51, \mathrm{p}=0.002$ between MSFsc and HbA1c in participants with more than 90 min SJL; Fig. 3); for the no, low, and moderate SJL groups there was no association between MSFsc and HbA1c levels. Notably, the range of the MSFsc in the four SJL groups was broadly the same (Supplementary Figure 3). Further, within the SJL>90 mins group, there was not a significant association between SJL and HbA1c $(r=-0.232, p=0.179)$, nor between SJL and MSFsc $(r=0.266, p=0.123)$ suggesting that the relationship between HbA1c and MSFsc in this group is not simply due to those participants with later MSFsc also significantly having more SJL. 


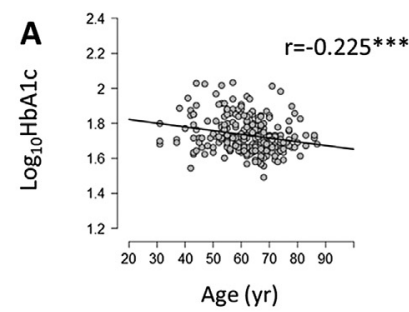

B

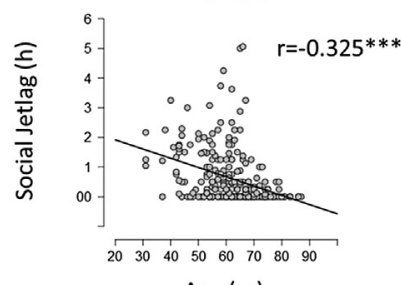

Age (yr)
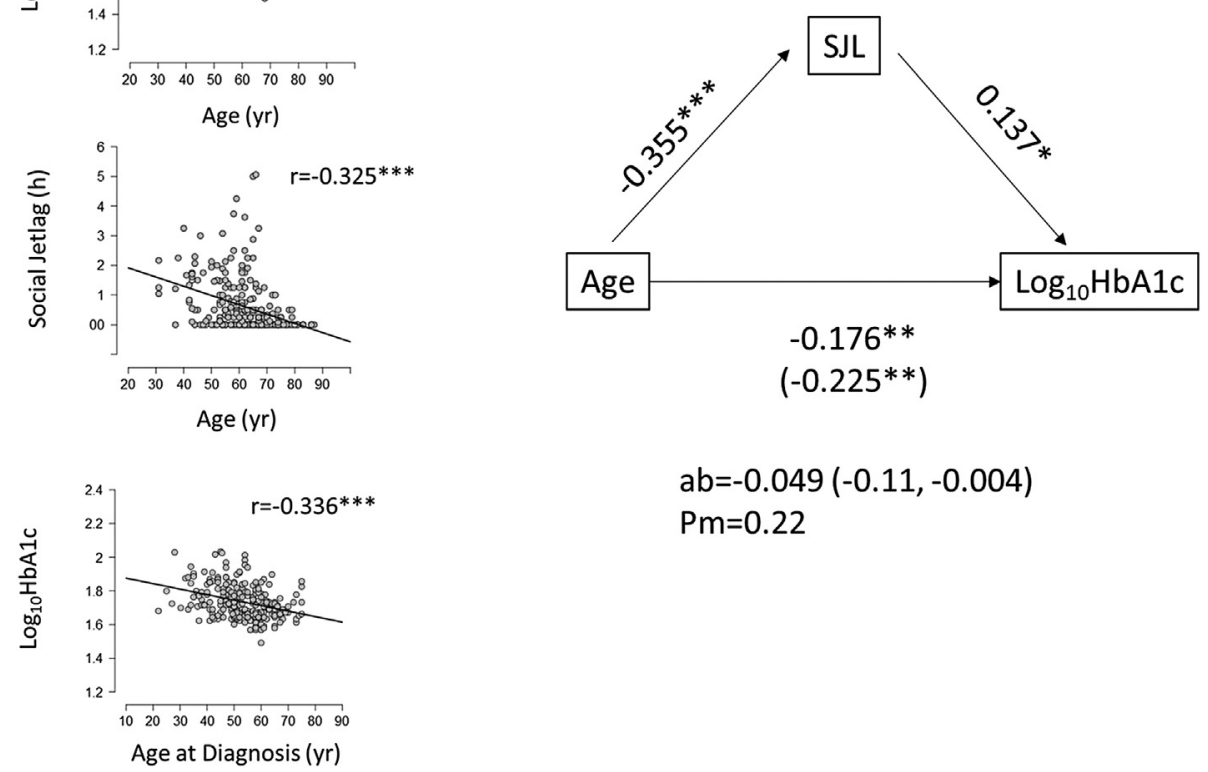

$a b=-0.049(-0.11,-0.004)$

$\mathrm{Pm}=0.22$

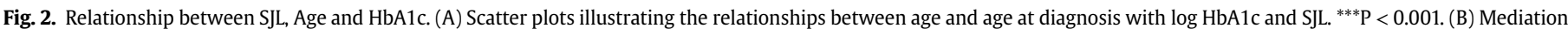

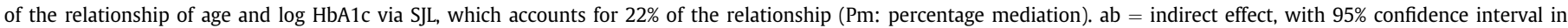
parentheses.

\section{Discussion}

In the present cohort of adults with type 2 diabetes, SJL was associated with glycemic control, independently of other predictors (Table 3). Later chronotype was associated with higher HbA1c only in participants with high levels of SJL, revealing a novel interaction between these two factors (Fig. 3). The relationship between chronotype and SJL that we report in this older adult cohort (mean age 61 years) is considerably weaker than previously reported in younger samples [8] suggesting that the majority of SJL in our cohort is due to social factors and that the effects of SJL and chronotype are mostly non-colinear. Therefore, the interaction observed between SJL and MSFsc in our cohort may be interpreted as an interplay of social factors and circadian characteristics. Our data provide support for the role of circadian misalignment in glycemic control in type 2 diabetes. There is some divergence between the current results and previous work on chronotype and glycemic control reporting an independent association between later chronotype (but not SJL) with poorer glycemic control in prediabetes and type 2 diabetes cohorts [12,14]. Identifying the nature of the social factors that shape SJL may provide novel psychosocial and psychoeducational targets to improve self-care in type 2 diabetes.

Currently, it remains unclear how chronotype and/or SJL may impact disease severity in type 2 diabetes. The relationship between the circadian clock and metabolism is likely to be bidirectional, as clock-dependent gene expression can be modulated by metabolic input, and metabolic functioning is profoundly affected by the circadian clock [1]. It is possible that at the whole organismlevel, later chronotype is associated with greater potential internal desynchrony in the distributed circadian system [35], which may lead to systemic pressures that result in poorer glycemic control or poorer response to medication in metabolic disease. Circadian misalignment reduces glucose tolerance and insulin sensitivity and increases levels of postprandial plasma glucose due to inadequate pancreatic $\beta$-cell function [36-38]. Following eight days of forced circadian misalignment, healthy participants displayed a prediabetic state of postprandial glucose responses [36]. Mistimed daytime sleep has been associated with metabolic disruption due to increased inflammation which may also explain how SJL may impair glucose metabolism [37]. Acute circadian misalignment has been associated with muscle-specific gene expression changes, demonstrating the relationship between circadian disruption, lower insulin-stimulated glucose disposal (a proxy measurement for muscle insulin sensitivity), elevated fasting blood glucose and free fatty acid levels [39]. Such changes may occur at the tissue and circulating level, with previous work showing various human plasma proteins involved in metabolic regulation being altered by circadian misalignment $[40,41]$.

In the present study sample most participants were overweight or obese. However, patients with high SJL did not have higher BMI than patients with no SJL (Fig. 1), and the association between SJL and HbA1c was independent of BMI (see Table 3). A previously reported association between chronotype and HbA1c in a type 2 diabetes sample was also independent of BMI [12]. No personality variables were associated with HbA1c in our regression model, suggesting that personality does not explain the associations between SJL or chronotype and glycemic control [12,14]. Further, while poorer glycemic control was associated with more depressive symptoms as well as insulin use, these variables did not differ between SJL groups. A potentially important factor that may play a role in mediating the relationship between SJL and glycemic control is work-related stress; in a typical type 2 diabetes population there will be individuals who are retired (who may have lower SJL due to diminished work-related demands on their sleep/wake behaviours), and those that are employed (who may experience greater 


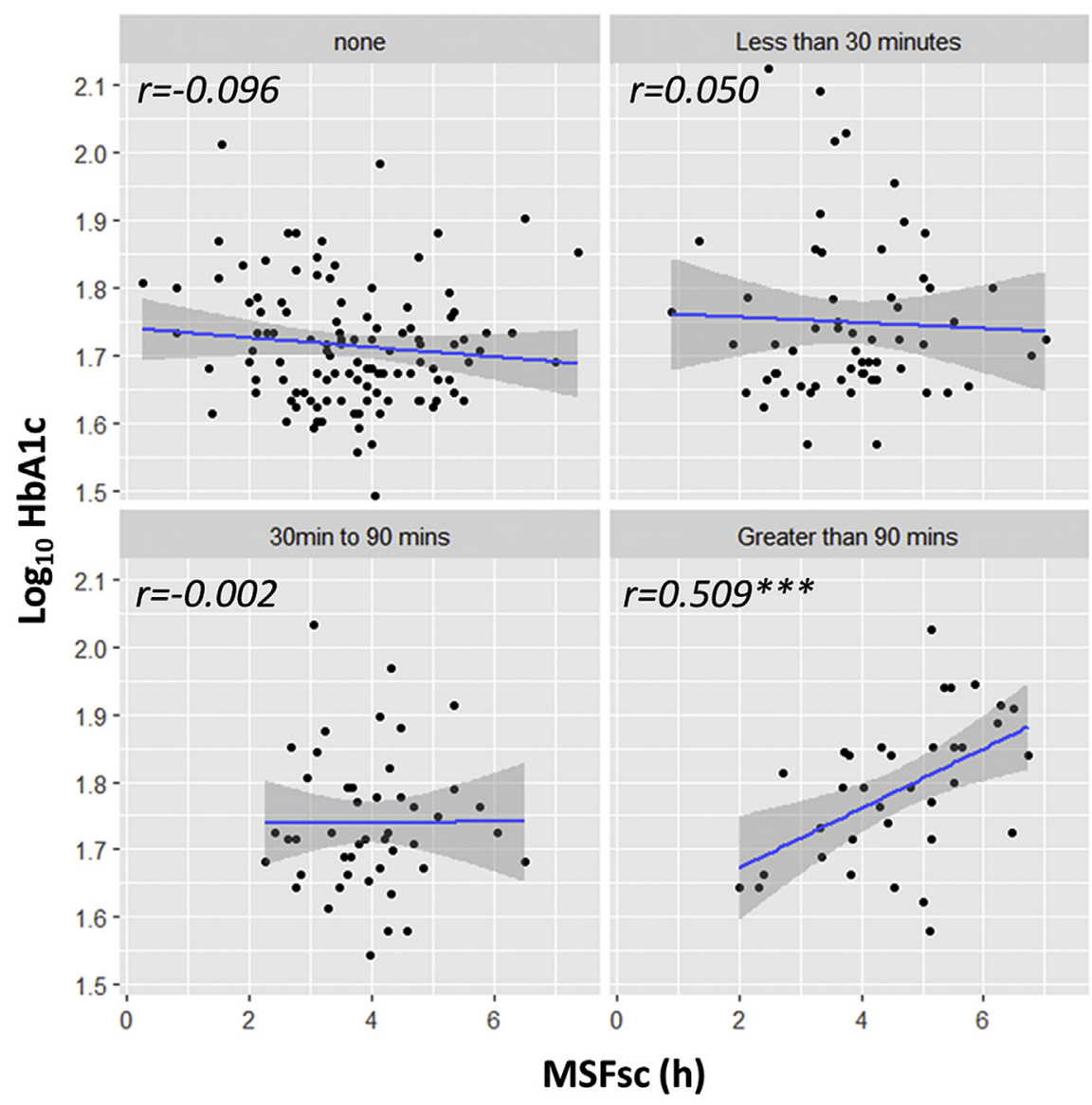

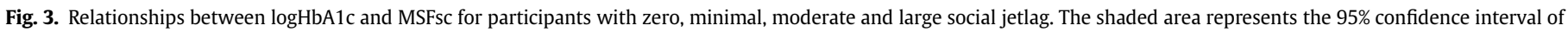
the regression line. Analyses by Pearson correlation. (None $\mathrm{n}=114$, less than $30 \min \mathrm{n}=57,30-90 \min \mathrm{n}=46$, greater than 90 min $\mathrm{n}=35$ ), ${ }^{* * *} \mathrm{P}<0.0001$.

SJL due to work scheduling). Those employed may experience greater levels of stress related to the workplace, which in turn may impact on disease severity [42]. However, there are also reports in the literature that workplace psychosocial stress does not impact on HbA1c levels in type 2 diabetes [43]. Future studies of SJL and glycemic control should address this possible relationship to address the question of whether SJL, in the context of glycemic control in type 2 diabetes, is a proxy measure for psychosocial stress, or is associated with poorer disease status independently of stress.

Short sleep duration accompanied by circadian misalignment may also be associated with decreased insulin sensitivity [44]. Further, as short sleep has consistently been reported as a risk factor for type 2 diabetes [45], SJL may be an important ancillary factor to consider in future studies of short sleep in type 2 diabetes. SJL may also involve wakefulness in the chronological morning but during individuals' biological night (eg, when individuals are awake but melatonin levels are still elevated) [46] which may potentially result in reduced insulin sensitivity and higher HbA1c. However, it is important to note that average sleep duration in our study had no relationship with HbA1c, suggesting that the effects we observed were as a result of circadian misalignment rather than shortened sleep (see Table 2). In experimental paradigms, oral glucose insulin sensitivity, but not intravenous insulin sensitivity, returns to baseline three days after the discontinuation of short sleep and circadian disruption suggesting differential effects and the potential for reversibility [40] (although recent data has called into question the efficacy of weekend "catch-up" sleep in ameliorating the metabolic effects of short sleep during the working week [47]).
A noteworthy and somewhat unexpected relationship between age, SJL and HbA1c emerged in our study (see Fig. 2). Younger age was associated with a higher HbA1c, mirroring recent reports suggesting more severe disease in younger adults with type 2 diabetes [48]. The group with the highest SJL in our study was also significantly younger than the individuals with no SJL. This finding is perhaps unsurprising given age-related differences in social imperatives and chronotype (younger age is associated with later mid-sleep). However, after controlling for age and years-sincediagnosis, HbA1c was still higher in those with more than 90 min SJL compared to those with no SJL. Further examination of the relationship between SJL, age and HbA1c via mediation analysis suggested that SJL may explain in the order of $20 \%$ of the effect of age on HbA1c in our sample. As such, SJL may represent an important topic of future research on disease outcomes in younger type 2 diabetes patients (taking into account the aforementioned caveats around potential roles for psychosocial stress). The mediation by SJL we report is concordant with other recent research which demonstrated that, for individuals 61 years of age or older, no association between SJL and diabetes prevalence was evident, but that individuals younger than 61 years of age who had more than $1 \mathrm{~h}$ SJL had a higher prevalence of diabetes than individuals with less than $1 \mathrm{~h}$ SJL [10].

\subsection{Strengths and limitations}

Whilst our study has strengths, including the replication of a previously published approach with minor modification [12] in a well-defined clinically-representative sample, there are important 
caveats and weaknesses to consider. First, due to the observational, cross-sectional nature of our study, the associations described should not be interpreted as causal. Our results suggest that SJL has a negative effect on glycemic control, but it is also possible that poor glycemic control influences SJL (although a mechanism for such a directional effect is not immediately apparent, but could be mediated through physiological sleep system changes due to altered glycemia). Second, it must be noted that the sample size of the participants in the group with the most SJL was smaller with only 35 people so this must be considered when interpreting the relationship observed here. Third, although our methodological approach is well-validated and replicates the methodology used previously, the measures of sleep, circadian, personality variables, OSA risk and dietary recall are subjective and/or rely on self-report, meaning that participants' memory and motivation play a big role. Objective measures may reveal additional layers of complexity and insight. Furthermore, the 24-h dietary recall may be biased via socially desirable responding towards over-reporting of more acceptable food choices. Further, calculating intake from a recall is only an estimate as while the databases are good for providing information on nutritional content, individuals may have different opinions of what a small or medium portion is, which could influence caloric estimates. Fourth, precise detail on food timing is required in future studies, with differences in intake on work and free days controlled for with a longer food log incorporating objective measures with time-stamps. This approach would increase confidence that SJL effects are not mediated through mealtiming and meal-content effects. Finally, we did not measure information on the participants work schedules beyond the number of free and work days per week. Information associated with work schedules including early work hours or late work evenings, and perceived work stress and other sources of psychosocial stress would be beneficial. Further analysis of other lifestyle factors, including physical activity, medication compliance and weight stability are required to examine if they may be playing a role in the relationship observed.

\section{Conclusions}

In summary, the results of our study demonstrate that greater SJL is associated with poorer glycemic control in patients with type 2 diabetes. Furthermore, we found a strong association between a later chronotype and poorer glycemic control in individuals with SJL of 90 minutes or greater. Of further interest, our results potentially suggest a greater deleterious effect of SJL on glycemic control in younger patients, and therefore that circadian factors may play a more important role in regulating metabolism in younger than in older adults with type 2 diabetes. Interventional studies to reduce SJL, particularly targeting younger individuals, may provide evidence of a cost-effective, non-pharmacologic intervention to improve type 2 diabetes disease management and decrease the occurrence of debilitating diabetes complications.

\section{Funding}

This research did not receive any specific grant from funding agencies in the public, commercial, or not-for-profit sectors.

\section{Acknowledgements}

No potential conflicts of interest to be noted. RK collected, researched and analysed the data, wrote, reviewed and edited the manuscript. JF, DG and UH collected data. JMcD, SS contributed to study design and the discussion. JMcD, SS and UH reviewed and edited the manuscript. AC contributed to the study design, analysed the data, contributed to the writing of the manuscript, reviewed and edited the manuscript. AC coordinated work between all authors involved in the study. RK/AC are the guarantors of this work.

\section{Conflict of interest}

The ICMJE Uniform Disclosure Form for Potential Conflicts of Interest associated with this article can be viewed by clicking on the following link: https://doi.org/10.1016/j.sleep.2019.07.023.

\section{Appendix A. Supplementary data}

Supplementary data to this article can be found online at https://doi.org/10.1016/j.sleep.2019.07.023.

\section{References}

[1] Panda S. Circadian physiology of metabolism. Science 2016;354(6315): 1008-15. https://doi.org/10.1126/science.aah4967.

[2] Roenneberg T, Kuehnle T, Juda M, et al. Epidemiology of the human circadian clock. Sleep Med Rev 2007;11(6):429-38. https://doi.org/10.1016/j.smrv. 2007.07.005.

[3] Di Milia L, Adan A, Natale V, et al. Reviewing the psychometric properties of contemporary circadian typology measures. Chronobiol Int 2013;30(10): 1261-71. https://doi.org/10.3109/07420528.2013.817415.

[4] Szosland D. Shift work and metabolic syndrome, diabetes mellitus and ischaemic heart disease. Int J Occup Med Environ Health 2010;23(3):287-91. https://doi.org/10.2478/v10001-010-0032-5.

[5] Gan Y, Yang C, Tong X, et al. Shift work and diabetes mellitus: a meta-analysis of observational studies. Occup Environ Med 2014;72(1):72-8. https://doi. org/10.1136/oemed-2014-102150.

[6] Sun M, Feng W, Wang F, et al. Meta-analysis on shift work and risks of specific obesity types. Obes Rev 2018;19(1):28-40. https://doi.org/10.1111/obr 12621.

[7] Wittmann M, Dinich J, Merrow M, et al. Social jetlag: misalignment of biological and social time. Chronobiol Int 2006;23(1-2):497-509. https://doi. org/10.1080/07420520500545979.

[8] Roenneberg T, Allebrandt KV, Merrow M, et al. Social jetlag and obesity. Curr Biol 2012;22(10):939-43. https://doi.org/10.1016/j.cub.2012.03.038.

[9] Wong PM, Hasler BP, Kamarck TW, et al. Social jetlag, chronotype, and cardiometabolic risk. J Clin Endocrinol Metab 2015;100(12):4612-20. https:// doi.org/10.1210/jc.2015-2923.

[10] Koopman ADM, Rauh SP, van 't Riet E, et al. The association between social jetlag, the metabolic syndrome, and type 2 diabetes mellitus in the general population: the New Hoorn Study. J Biol Rhythm 2017;32(4):359-68. https:/ doi.org/10.1177/0748730417713572.

[11] Parsons MJ, Moffitt TE, Gregory AM, et al. Social jetlag, obesity and metabolic disorder: investigation in a cohort study. Int J Obes (Lond) 2015;39(5):842-8. https://doi.org/10.1038/ijo.2014.201.

[12] Reutrakul S, Hood MM, Crowley SJ, et al. Chronotype is independently associated with glycemic control in type 2 diabetes. Diabetes Care 2013;36(9): 2523-9. https://doi.org/10.2337/dc12-2697.

[13] Reutrakul S, Hood MM, Crowley SJ, et al. The relationship between breakfast skipping, chronotype, and glycemic control in type 2 diabetes. Chronobiol Int 2014;31(1):64-71. https://doi.org/10.3109/07420528.2013.821614.

[14] Anothaisintawee T, Lertrattananon D, Thamakaison S, et al. Later chronotype is associated with higher hemoglobin A1c in prediabetes patients. Chronobio Int 2017;34(3):393-402. https://doi.org/10.1080/07420528.2017.1279624.

[15] Nimitphong $H$, Siwasaranond N, Saetung S, et al. The relationship among breakfast time, morningness-eveningness preference and body mass index in type 2 diabetes. Diabet Med 2018;35(7):964-71. https://doi.org/10.1111/ dme.13642.

[16] Brown AW, Bohan Brown MM, Allison DB. Belief beyond the evidence: using the proposed effect of breakfast on obesity to show 2 practices that distort scientific evidence. Am J Clin Nutr 2013;98(5):1298-308. https://doi.org/10. 3945/ajcn.113.064410.

[17] Jakubowicz D, Wainstein J, Ahren B, et al. Fasting until noon triggers increased postprandial hyperglycemia and impaired insulin response after lunch and dinner in individuals with type 2 diabetes: a randomized clinical trial. Diabetes Care 2015;38(10):1820-6. https://doi.org/10.2337/dc15-0761.

[18] Mota MC, Silva CM, Balieiro LC, et al. Social jetlag and metabolic control in non-communicable chronic diseases: a study addressing different obesity statuses. Sci Rep 2017:7:6358. https://doi.org/10.1038/s41598-017-06723-w.

[19] Larcher S, Gauchez AS, Lablanche S, et al. Impact of sleep behavior on glycemic control in type 1 diabetes: the role of social jetlag. Eur $\mathrm{J}$ Endocrinol 2016;175(5):411-9. https://doi.org/10.1530/EJE-16-0188.

[20] Von Schnurbein J, Boettcher C, Brandt S, et al. Sleep and glycemic control in adolescents with type 1 diabetes. Pediatr Diabetes 2018;19(1):143-9. https:/| doi.org/10.1111/pedi.12538. 
[21] Mokhlesi B, Temple KA, Tjaden AH, et al. Association of self-reported sleep and circadian measures with glycemia in adults with prediabetes or recently diagnosed untreated type 2 diabetes. Diabetes Care 2019;42(7):1326-32. https://doi.org/10.2337/dc19-0298.

[22] Adan A, Archer SN, Hidalgo MP, et al. Circadian typology: a comprehensive review. Chronobiol Int 2012;29(9):1153-75. https://doi.org/10.3109/ 07420528.2012.719971.

[23] Lipnevich AA, Credè M, Hahn E, et al. How distinctive are morningness and eveningness from the Big Five factors of personality? A meta-analytic investigation. J Personal Soc Psychol 2017;112(3):491-509. https://doi.org/10. 1037/pspp0000099.

[24] Skinner TC, Bruce DG, Davis TM, et al. Personality traits, self-care behaviours and glycaemic control in type 2 diabetes: the Fremantle Diabetes Study Phase II. Diabet Med 2014;31(4):487-92. https://doi.org/10.1111/dme.12339.

[25] Snoek FJ. Psychological aspects of diabetes management. Medicine 2002:30(1):14-5. https://doi.org/10.1383/medc.30.1.14.28265.

[26] Silva CM, Mota MC, Miranda MT, et al. Chronotype, social jetlag and sleep debt are associated with dietary intake among Brazilian undergraduate students. Chronobiol Int 2016;33(6):740-8. https://doi.org/10.3109/07420528.2016. 1167712.

[27] Mota MC, Silva CM, Balieiro LC, et al. Association between social jetlag food consumption and meal times in patients with obesity-related chronic diseases. PLoS One 2019;14(2):e0212126. https://doi.org/10.1371/journal.pone. 0212126.

[28] Tabachnick BG, Fidell LS. Using multivariate statistics. 6th ed. International edition: Allyn \& Bacon/Pearson Education; 2013.

[29] Roenneberg T, Wirz-Justice A, Merrow M. Life between clocks: daily temporal patterns of human chronotypes. J Biol Rhythm 2003;18(1):80-90. https://doi. org/10.1177/0748730402239679.

[30] Radloff LS. The CES-D Scale: a self-report depression scale for research in the general population. Appl Psychol Meas 1977;1(3):385-401.

[31] John OP, Srivastava S. The Big Five trait taxonomy: history, measurement, and theoretical perspectives. In: Pervin LA, John OP, editors. Handbook of personality: theory and research. New York: Guilford Press; 1999. p. 102-38.

[32] Buysse DJ, Reynolds Iii CF, Monk TH, et al. The Pittsburgh Sleep Quality Index: a new instrument for psychiatric practice and research. Psychiatry Res 1989;28(2):193-213.

[33] Netzer NC, Stoohs RA, Netzer CM, et al. Using the Berlin Questionnaire to identify patients at risk for the sleep apnea syndrome. Ann Intern Med 1999;131(7):485-91.

[34] Hayes AF. Introduction to mediation, moderation, and conditional process analysis: a regression-based approach. 2nd ed. New York, NY: The Guilford Press; 2017.

[35] Roenneberg T, Merrow M. The circadian clock and human health. Curr Biol 2016;26(10):R432-43. https://doi.org/10.1016/j.cub.2016.04.011.
[36] Scheer FA, Hilton MF, Mantzoros CS, et al. Adverse metabolic and cardiovascular consequences of circadian misalignment. Proc Natl Acad Sci USA 2009;106(11):4453-8. https://doi.org/10.1073/pnas.0808180106.

[37] Leproult R, Holmbäck U, Van Cauter E. Circadian misalignment augments markers of insulin resistance and inflammation, independently of sleep loss. Diabetes 2014;63(6):1860-9. https://doi.org/10.2337/db13-1546.

[38] Morris CJ, Yang JN, Garcia JI, et al. Endogenous circadian system and circadian misalignment impact glucose tolerance via separate mechanisms in humans. Proc Natl Acad Sci USA 2015;112(17):E2225-34. https://doi.org/10.1073/ pnas. 1418955112.

[39] Wefers J, van Moorsel D, Hansen J, et al. Circadian misalignment induces fatty acid metabolism gene profiles and compromises insulin sensitivity in human skeletal muscle. Proc Natl Acad Sci USA 2018;115(30):7789-94. https://doi. org/10.1073/pnas.1722295115.

[40] Cedernaes J, Osler ME, Voisin S, et al. Acute sleep loss induces tissue-specific epigenetic and transcriptional alterations to circadian clock genes in men. JCEM 2015;100(9):E1255-61. https://doi.org/10.1210/JC.2015-2284.

[41] Depner CM, Melanson EL, McHill AW, et al. Mistimed food intake and sleep alters 24-hour time-of-day patterns of the human plasma proteome. Proc Natl Acad Sci USA 2018;115(23):E5390-9. https://doi.org/10.1073/pnas. 1714813115.

[42] Kawakami N, Akachi K, Shimizu H, et al. Job strain, social support in the workplace, and haemoglobin A1c in Japanese men. Occup Environ Med 2000;57(12):805-9. https://doi.org/10.1136/oem.57.12.805.

[43] Annor FB, Roblin DW, Okosun IS, et al. Work-related psychosocial stress and glycemic control among working adults with diabetes mellitus. Diabetes Metab Syndrome 2015;9(2):85-90. https://doi.org/10.1016/j.dsx.2015.02. 003.

[44] Eckel RH, Depner CM, Perreault L, et al. Morning circadian misalignment during short sleep duration impacts insulin sensitivity. Curr Biol 2015;25(22): 3004-10. https://doi.org/10.1016/j.cub.2015.10.011.

[45] Yadav D, Cho KH. Total sleep duration and risk of type 2 diabetes: evidence based on clinical and epidemiological studies. Curr Drug Metabol 2018;29(6): 957-67. https://doi.org/10.2174/1389200219666180628170431.

[46] Wright Jr KP, McHill AW, Birks BR, et al. Entrainment of the human circadian clock to the natural light-dark cycle. Curr Biol 2013;23(16):1554-8. https:// doi.org/10.1016/j.cub.2013.06.039.

[47] Depner CM, Melanson EL, Eckel RH, et al. Ad libitum weekend recovery sleep fails to prevent metabolic dysregulation during a repeating pattern of insufficient sleep and weekend recovery sleep. Curr Biol 2019;29(6):957-67. https://doi.org/10.1016/j.cub.2019.01.069.

[48] Shamshirgaran SM, Mamaghanian A, Aliasgarzadeh A, et al. Age differences in diabetes-related complications and glycemic control. BMC Endocr Disord 2017;17(1):25. https://doi.org/10.1186/s12902-017-0175-5. 